\title{
DYNAMICS FOR GINZBURG-LANDAU VORTICES UNDER A MIXED FLOW
}

\author{
MATTHIAS KURZKE, CHRISTOF MELCHER, ROGER MOSER, \\ AND DANIEL SPIRN
}

\begin{abstract}
We consider a complex Ginzburg-Landau equation that contains a Schrödinger term and a damping term that is proportional to the time derivative. Given well-prepared initial conditions that correspond to quantized vortices, we establish the vortex motion law until collision time.
\end{abstract}

\section{INTRODUCTION}

We study the asymptotic behavior of the complex Ginzburg-Landau equation

$$
\begin{aligned}
\left(\alpha_{\varepsilon}+i\right) \partial_{t} u_{\varepsilon} & =\Delta u_{\varepsilon}+\frac{1}{\varepsilon^{2}} u_{\varepsilon}\left(1-\left|u_{\varepsilon}\right|^{2}\right) \\
u_{\varepsilon}(\cdot, 0) & =u_{\varepsilon}^{0}(\cdot)
\end{aligned}
$$

as $\varepsilon \rightarrow 0$, where $u_{\varepsilon}(t): \Omega \rightarrow \mathbb{C}, \Omega \subset \mathbb{R}^{2}$ a bounded and simply connected $C^{1}$ domain, with either Dirichlet boundary conditions

$$
u_{\varepsilon}=g \text { on } \partial \Omega,
$$

where $g \in C^{1}\left(\partial \Omega, S^{1}\right)$, or Neumann boundary conditions,

$$
\frac{\partial u_{\varepsilon}}{\partial \nu}=0 \quad \text { on } \partial \Omega
$$

The flow used in (1.1) is a hybrid between the time-rescaled gradient flow

$$
\alpha_{\varepsilon} \partial_{t} u_{\varepsilon}=\Delta u_{\varepsilon}+\frac{1}{\varepsilon^{2}} u_{\varepsilon}\left(1-\left|u_{\varepsilon}\right|^{2}\right)
$$

and the Schrödinger flow (also known as Gross-Pitaevskii equation from the theory of superfluidity)

$$
i \partial_{t} u_{\varepsilon}=\Delta u_{\varepsilon}+\frac{1}{\varepsilon^{2}} u_{\varepsilon}\left(1-\left|u_{\varepsilon}\right|^{2}\right)
$$

Date: November 4, 2008.

2000 Mathematics Subject Classification. Primary: 35B20, Secondary: 35Q55, 35B40.

The research presented in this article was done while the authors enjoyed the hospitality of the Hausdorff Research Institute for Mathematics, Bonn. MK was supported by DFG SFB 611. DS was supported by NSF grant DMS-0707714. 
The right-hand side is in both cases the negative $L^{2}$ gradient of the GinzburgLandau energy

$$
E_{\varepsilon}(u)=\frac{1}{2} \int_{\Omega}|\nabla u|^{2}+\frac{1}{2 \varepsilon^{2}}\left(1-|u|^{2}\right)^{2}=\int_{\Omega} e_{\varepsilon}(u) .
$$

Both (1.5) and (1.6) have been widely studied in the literature. Formal results using matched asymptotics were obtained by Neu [19] and E [9]; the rigorous literature starts with Lin [14, 15] and Jerrard-Soner [11] for the gradient flow and Colliander-Jerrard [6,7] and Lin-Xin [17] for the Schrödinger equation. For subsequent refinements we mention, among other works, Bethuel-Orlandi-Smets [4,5] and Serfaty [23] for the gradient flow and Jerrard-Spirn [12] for the Schrödinger case.

The equation (1.1) can be seen as a damped Schrödinger equation where the damping is proportional to the rate of change in the wave function. Our research into this mixed flow is motivated by the formal analogy between (1.1) and the Landau-Lifshitz-Gilbert equations for the dynamics of a ferromagnetic thin film with a strong in-plane anisotropy:

$$
\mathbf{m}_{t}+\alpha \mathbf{m} \times \mathbf{m}_{t}=\gamma \mathbf{m} \times\left(\Delta \mathbf{m}-\frac{1}{\varepsilon^{2}} m_{3} \mathbf{e}_{3}\right),
$$

where $\mathbf{m}: \Omega \rightarrow S^{2}$ and $\mathbf{e}_{3}$ is the unit vector orthogonal to the film plane. The analogy between (1.8) and (1.1) becomes clear by multiplying from the left with $\mathbf{m} \times$ and identifying the in-plane rotation $v \mapsto i v$ with the rotation around the magnetization vector, $\mathbf{h} \mapsto \mathbf{m} \times \mathbf{h}$. We establish a vortex motion law for (1.8) in [13]. Some previous results in this direction are due to Hang-Lin [10], who studied the static problem, and to Lin-Shatah [16], who studied the motion law for undamped vortex motion following the approach of Lin-Xin [17].

As an independent additional motivation, we note that (1.1) results from the time-dependent Ginzburg-Landau equation describing the behavior of superconductors if one omits the gauge term. The strictly complex character of the relaxation constant has been used in the physics literature to explain the sign of the Hall effect in type II superconductors, see the work of Dorsey [8]. A vortex motion law for this case was derived using matched asymptotics by E [9].

We will study $\alpha_{\varepsilon}$ corresponding to the gradient flow time scale

$$
\alpha_{\varepsilon}|\log \varepsilon| \rightarrow \alpha_{0} \in(0, \infty) \quad \text { as } \varepsilon \rightarrow 0
$$

and will only consider well-prepared initial data, meaning initial data that are asymptotically minimizing given a limit vorticity.

Equation (1.1) with this choice of timescales appears as a side note in [17, Section 6], where the authors' interest is to show uniform continuity of vortex motions independent of small $\alpha_{0}$. Our results shed some further light on arguments such as these, and in particular we give the exact motion law before the limit $\alpha_{0} \rightarrow 0$. 
It is perhaps interesting to note that the linear combination of the nonlinear PDEs (1.5) and (1.6) yields a linear combination of the corresponding nonlinear ODEs for the vortex dynamics. For well-prepared initial data that corresponds to vortices of degree $d_{i} \in\{ \pm 1\}$ at distinct points $a_{i} \in \Omega$, (1.5) gives rise to the vortex motion law

$$
\left(\begin{array}{cc}
\alpha_{0} & 0 \\
0 & \alpha_{0}
\end{array}\right) \dot{a}_{i}=-\frac{1}{\pi} \nabla_{a_{i}} W(a, d),
$$

while (1.6) leads to

$$
\left(\begin{array}{cc}
0 & -d_{i} \\
d_{i} & 0
\end{array}\right) \dot{a}_{i}=-\frac{1}{\pi} \nabla_{a_{i}} W(a, d),
$$

where the function $W$ is in both cases the renormalized energy of [1].

For (1.1), we can now formally obtain a mixed motion law by just adding the left hand sides of (1.10) and (1.11), yielding

$$
\left(\begin{array}{cc}
\alpha_{0} & -d_{i} \\
d_{i} & \alpha_{0}
\end{array}\right) \dot{a}_{i}=-\frac{1}{\pi} \nabla_{a_{i}} W(a, d) .
$$

The main result of this article, Theorem 2.2, establishes that this completely formal calculation indeed gives the correct result, so the dynamical law (1.12) holds true for vortices of degree \pm 1 until either two vortices collide or (in the case of Neumann boundary conditions) a vortex leaves the domain. The time when that happens can be calculated just using the limiting ODE.

Our approach uses a combination of methods used for gradient flows (mainly, the kinetic energy bound of Sandier-Serfaty [21]) and methods that were developed for the Schrödinger equation, in particular the results of LinXin [17], Colliander-Jerrard [6, 7] and Jerrard-Spirn [12]. The main new ingredient in our proof is to use that both the Jacobian and the rescaled energy density converge to the same limit, and we can therefore study linear combinations of the conservation laws for these quantities with suitable test functions that have good cancellation properties near the vortex core.

Independent from our research, Miot [18] has established a result complementary to ours for the case of an unbounded domain, where the energy is infinite unless the total degree is 0 .

\section{Statement of the MAin RESUlt}

To state our main result in a precise way, we need to introduce some notation. For complex numbers $z, w$ we use the real-valued inner product corresponding to the identification of $\mathbb{C}$ with $\mathbb{R}^{2}:(z, w)=\frac{1}{2}(\bar{z} w+z \bar{w})$. By $\mathbb{J}$, we denote the symplectic matrix given by

$$
\mathbb{J}=\left(\begin{array}{cc}
0 & 1 \\
-1 & 0
\end{array}\right) \text {. }
$$


We assume that $\Omega$ is a bounded and simply connected domain in $\mathbb{R}^{2}$ with $C^{1}$ boundary. For $a=\left(a_{1}, \ldots, a_{n}\right) \in \Omega^{n}$, we define

$$
\rho_{a}=\frac{1}{4} \min \left\{\min _{i \neq j}\left|a_{i}-a_{j}\right|, \min _{i} \operatorname{dist}\left(a_{i}, \partial \Omega\right)\right\}
$$

and, for $s<\rho_{a}$, the corresponding domains with holes punched out,

$$
\Omega_{s}(a)=\Omega \backslash \bigcup_{i=1}^{n} \overline{B_{s}\left(a_{i}\right)} .
$$

We use the following weak norm on the dual of $W_{0}^{1, p}(\Omega)$ :

$$
\|\mu\|_{\dot{W}^{-1, q}}=\sup \left\{\mu(\phi): \phi \in W_{0}^{1, p}(\Omega),\|\nabla \phi\|_{L^{p}} \leq 1\right\}, \quad \frac{1}{p}+\frac{1}{q}=1 .
$$

The corresponding space-time norms will be denoted by subscripts $t$ and $x$.

We note that the $\dot{W}^{-1,1}$ norm corresponds to the length of a minimal connection in the terminology of Brezis-Coron-Lieb [2]; in particular, for $a \in \Omega^{n}, \rho_{a}>0, \xi \in \Omega^{n}$ with $\max _{i}\left|\xi_{i}-a_{i}\right| \leq \rho_{a}$,

$$
\left\|\pi \sum_{i=1}^{n} d_{i}\left(\delta_{a_{i}}-\delta_{\xi_{i}}\right)\right\|_{\dot{W}^{-1,1}}=\pi \sum_{i=1}^{n}\left|d_{i}\right|\left|\xi_{i}-a_{i}\right| .
$$

For $a \in \Omega^{n}$ with $\rho_{a}>0$ and $d \in \mathbb{Z}^{n}$, the canonical harmonic map $u_{*}=$ $u_{*}^{(a, d)}$ is defined as an $\mathbb{S}^{1}$-valued map that solves the equations

$$
\begin{aligned}
\operatorname{div} j\left(u_{*}\right) & =0 \\
\operatorname{curl} j\left(u_{*}\right) & =2 \pi \sum_{i=1}^{n} d_{i} \delta_{a_{i}}
\end{aligned}
$$

with either Dirichlet boundary conditions $u_{*}=g$ or Neumann boundary conditions $\nu \cdot j\left(u_{*}\right)=0$. This defines the canonical harmonic map uniquely (in the Dirichlet case) or up to a constant (in the Neumann case), see [1] for more details.

We define the corresponding renormalized energy as

$$
W(a, d)=\lim _{\rho \rightarrow 0}\left(\int_{\Omega_{\rho}(a)} \frac{1}{2}\left|\nabla u_{*}\right|^{2}-\pi \sum_{i=1}^{n} d_{i}^{2} \log \frac{1}{\rho}\right) .
$$

We note that $u_{*}$ is the unique minimizer of the Dirichlet energy under the condition (2.7), up to the limit process inherent in (2.8).

Our notation does not show the dependence on $\Omega, g$, or whether we choose Dirichlet or Neumann boundary conditions.

The renormalized energy and the full Ginzburg-Landau energy $E_{\varepsilon}$ are connected via the following proposition, which is essentially the lower bound part of a second order $\Gamma$-convergence: 
Proposition 2.1. Assume that $\left(u_{\varepsilon}\right)$ is a sequence of functions with $J\left(u_{\varepsilon}\right) \rightarrow$ $\pi \sum_{i=1}^{n} d_{i} \delta_{a_{i}}, d_{i} \in\{ \pm 1\}, a_{i} \in \Omega, \rho_{a}>0$. Then

$$
\liminf _{\varepsilon \rightarrow 0}\left(E_{\varepsilon}\left(u_{\varepsilon}\right)-\pi n|\log \varepsilon|\right) \geq n \gamma+W(a, d),
$$

where $W(a, d)$ denotes the Dirichlet renormalized energy if all $u_{\varepsilon}$ satisfy $u_{\varepsilon}=g$ on $\partial \Omega$, and the Neumann renormalized energy otherwise, and $\gamma$ is defined via

$$
\gamma=\lim _{\varepsilon \rightarrow 0}\left(\inf \left\{\int_{B_{1}(0)} e_{\varepsilon}(u): u(z)=\frac{z}{|z|} \text { on } \partial B_{1}(0)\right\}-\pi|\log \varepsilon|\right),
$$

where $e_{\varepsilon}(u)$ is defined in (1.7).

Proof. The existence of the limit for $\gamma$ was shown in [1, Lemma IX.1]. The full result of (2.9) was shown by Serfaty [24, 25], and a more quantitative analysis can be found in [12].

The last proposition motivates the following definition: the approximate energy is given by

$$
W_{\varepsilon}(a, d)=n(\pi|\log \varepsilon|+\gamma)+W(a, d) .
$$

We define some useful quantities for sufficiently smooth functions $u$ : the energy density

$$
e_{\varepsilon}(u)=\frac{1}{2}|\nabla u|^{2}+\frac{1}{4 \varepsilon^{2}}\left(1-|u|^{2}\right)^{2},
$$

the current

$$
j(u)=(i u, \nabla u)
$$

the vorticity or Jacobian

$$
J(u)=\frac{1}{2} \operatorname{curl} j(u)=\operatorname{det} \nabla u,
$$

where in the last equation, we have used the identification of $\mathbb{C}$ and $\mathbb{R}^{2}$.

We now have all the notation in place to state our main result:

Theorem 2.2. Let $\left(u_{\varepsilon}\right): \Omega \times\left[0, T_{0}\right) \rightarrow \mathbb{C}, 0<T_{0} \leq \infty$ be a sequence of solutions of (1.1) such that the initial data $u_{\varepsilon}^{0}$ of (1.2) satisfy

$$
\left\|J\left(u_{\varepsilon}^{0}\right)-\pi \sum_{i=1}^{n} d_{i} \delta_{a_{i}^{0}}\right\|_{\dot{W}^{-1,1}} \rightarrow 0 \quad \text { as } \varepsilon \rightarrow 0
$$

for some $d_{i} \in\{ \pm 1\}, a_{i} \in \Omega, a_{i} \neq a_{j}$ for $i \neq j$, and

$$
E_{\varepsilon}\left(u_{\varepsilon}^{0}\right)-W_{\varepsilon}(a, d) \rightarrow 0 \quad \text { as } \varepsilon \rightarrow 0,
$$

where $W_{\varepsilon}$ is defined in (2.11). 
Then if the time scale is chosen as in (1.9), the rescaled energy density $\frac{\alpha_{\varepsilon}}{\alpha_{0}} e_{\varepsilon}\left(u_{\varepsilon}(\cdot, t)\right)$ converges to a measure $\mu(t)$. The Jacobians $J\left(u_{\varepsilon}(\cdot, t)\right)$ converge to a measure $\nu(t)$ such that $|\nu(t)|=\mu(t)$ and the measure $\nu(t)$ can be written as

$$
\nu(t)=\pi \sum_{i=1}^{n} d_{i} \delta_{a_{i}(t)},
$$

where the points $a(t)=\left(a_{i}(t)\right)$ satisfy the motion law

$$
\alpha_{0} \dot{a}_{i}-d_{i} \rrbracket \dot{a}_{i}=-\frac{1}{\pi} \nabla_{a_{i}} W(a(t), d)
$$

or equivalently,

$$
\dot{a}_{i}=-\frac{1}{\pi\left(\alpha_{0}^{2}+1\right)}\left(\begin{array}{cc}
\alpha_{0} & d_{i} \\
-d_{i} & \alpha_{0}
\end{array}\right) \nabla_{a_{i}} W(a(t), d)
$$

with initial condition

$$
a_{i}(0)=a_{i}^{0}
$$

for all $t<T=\min \left(T_{0}, T_{1}\right)$, where $T_{1}$ is the maximal time of existence for the $O D E$ (2.17) and $W$ is the renormalized energy given in (2.8).

Furthermore, $\lim _{\varepsilon \rightarrow 0}\left(E_{\varepsilon}\left(u_{\varepsilon}(t)\right)-W_{\varepsilon}(a(t), d)\right)=0$ for all $t \in[0, T)$, so the well-preparedness of the initial data stays true for all times.

Remark 2.3. The existence of initial data such that (2.15) and (2.16) hold is shown e.g. in [12, Lemma 14].

Remark 2.4. The existence and regularity for solutions of (1.1) is essentially standard. For $\varepsilon$ fixed, (1.1) can be considered as a semilinear parabolic $2 \times 2$ system $\partial_{t} u+L u=N(u)$ where $L$ is strictly elliptic and $N=N(u)$ cubic. By Galerkin's method, we infer that there exists a global weak solution $u \in \dot{H}_{t}^{1} L_{x}^{2} \cap L_{t}^{\infty} H_{x}^{1}$. Moreover, by Sobolev embedding, $u \in L_{\mathrm{loc}}^{p}$ and thus by parabolic (Calderon-Zygmund) a priori estimates $\partial_{t} u, D^{2} u \in L_{\mathrm{loc}}^{p}$ for every $p<\infty$, so the solution will be regular. We note that $L^{p}$ regularity of $\nabla u(t)$ holds up to the boundary of $\Omega$.

\section{Conservation LAWS AND RELATED identities}

In this section, we define some more quantities and establish several evolution identities. The solutions $u_{\varepsilon}$ are sufficiently regular to allow our manipulations by Remark 2.4 .

Deviating from the nomenclature used in the context of the Gross-Pitaevskii equation (where $j$ is sometimes called "momentum" as it describes the velocity field of a superfluid), we define the momentum as

$$
p(u)=\left(\partial_{t} u, \nabla u\right) .
$$

The stress-energy tensor $\mathrm{T}^{\varepsilon}$ is given by

$$
\mathbf{T}_{i j}^{\varepsilon}(u)=\left(\partial_{i} u, \partial_{j} u\right)-\delta_{i j} e_{\varepsilon}(u) .
$$


As an abbreviation, we write $f_{\varepsilon}=f_{\varepsilon}\left(u_{\varepsilon}\right)$ for the right-hand side of (1.1), which is the negative $L^{2}$ gradient of the energy (1.7):

$$
f_{\varepsilon}(u)=\Delta u+\frac{1}{\varepsilon^{2}} u\left(1-|u|^{2}\right) .
$$

The importance of the stress-energy tensor is seen in the following stressenergy identity of Pohožaev type:

$$
\left.\left(f_{\varepsilon}(u), \partial_{i} u\right)=\partial_{j} \mathbf{T}_{i j}^{\varepsilon}(u)\right)
$$

where we have used the convention of summing over double indices. We also note that

$$
\operatorname{curl} \operatorname{div} \mathrm{T}(u)=\operatorname{curl} \operatorname{div}(\nabla u \otimes \nabla u)
$$

so

$$
\operatorname{curl}\left(f_{\varepsilon}(u), \nabla u\right)=\mathbb{J}_{i j} \partial_{i} \partial_{k}\left(\partial_{k} u, \partial_{j} u\right) .
$$

From the identity

$$
\partial_{t}(i u, \nabla u)-\nabla\left(i u, \partial_{t} u\right)=2\left(i \partial_{t} u, \nabla u\right)
$$

we obtain, by taking the curl, an identity for the time-evolution of $J(u)$

$$
\partial_{t} J(u)=\operatorname{curl}\left(i \partial_{t} u, \nabla u\right) \text {. }
$$

From the evolution equation (1.1) we can derive some conservation laws. Multiplying the equation by $i u_{\varepsilon}$, we obtain the conservation of mass equation

$$
\partial_{t}\left(\frac{1}{2}\left(\left|u_{\varepsilon}\right|^{2}-1\right)\right)=\operatorname{div} j\left(u_{\varepsilon}\right)-\alpha_{\varepsilon}\left(i u_{\varepsilon}, \partial_{t} u_{\varepsilon}\right) .
$$

Testing the equation with $\nabla u_{\varepsilon}$ and using the relation $\partial_{t}(i u, \nabla u)-\nabla\left(i u, \partial_{t} u\right)=$ $2\left(i \partial_{t} u, \nabla u\right)$, we obtain after some algebra the conservation law for the current:

$$
\partial_{t} j\left(u_{\varepsilon}\right)=2 \operatorname{div}\left(\nabla u_{\varepsilon} \otimes \nabla u_{\varepsilon}\right)-\nabla P_{\varepsilon}-2 \alpha_{\varepsilon} p\left(u_{\varepsilon}\right),
$$

where the pressure $P_{\varepsilon}$ is given by

$$
P_{\varepsilon}=\left|\nabla u_{\varepsilon}\right|^{2}+\left(u_{\varepsilon}, \Delta u_{\varepsilon}\right)-\frac{\left|u_{\varepsilon}\right|^{4}-1}{2 \varepsilon^{2}}-\alpha_{\varepsilon}\left(u_{\varepsilon}, \partial_{t} u_{\varepsilon}\right) .
$$

It follows that the Jacobian satisfies

$$
\partial_{t} J\left(u_{\varepsilon}\right)=\operatorname{curl} \operatorname{div}\left(\nabla u_{\varepsilon} \otimes \nabla u_{\varepsilon}\right)-\alpha_{\varepsilon} \operatorname{curl} p\left(u_{\varepsilon}\right) .
$$

Testing (1.1) with $\partial_{t} u_{\varepsilon}$ yields the conservation law for the energy density,

$$
\partial_{t} e_{\varepsilon}\left(u_{\varepsilon}\right)=-\alpha_{\varepsilon}\left|\partial_{t} u_{\varepsilon}\right|^{2}+\operatorname{div} p\left(u_{\varepsilon}\right) .
$$

On the left-hand side of both (3.13) and (3.12), we have expressions related to the vortex velocity, since both $J\left(u_{\varepsilon}\right)$ and $\frac{1}{|\log \varepsilon|} e_{\varepsilon}\left(u_{\varepsilon}\right)$ converge to measures supported on the vortex sites. At first glance, both equations appear to be difficult to use without detailed control of the momentum $p\left(u_{\varepsilon}\right)$, 
which does not seem to be easy to obtain. However, we can get rid of the influence of $p\left(u_{\varepsilon}\right)$ at the vortex site by combining equations for energy density and Jacobian with suitable test functions.

Proposition 3.1. For any $\phi, \psi \in W_{0}^{2, \infty}(\Omega)$, we have the following evolution equation for linear combinations of $e_{\varepsilon}\left(u_{\varepsilon}\right)$ and $J\left(u_{\varepsilon}\right)$ :

$$
\begin{aligned}
\partial_{t} \int_{\Omega}\left(\alpha_{\varepsilon} \psi e_{\varepsilon}\left(u_{\varepsilon}\right)\right. & \left.+\phi J\left(u_{\varepsilon}\right)\right) \\
= & \frac{1}{\alpha_{\varepsilon}^{2}+1} \int_{\Omega} \mathbb{J}_{i j} \partial_{i} \partial_{k} \phi\left(\partial_{k} u_{\varepsilon}, \partial_{j} u_{\varepsilon}\right) \\
-\alpha_{\varepsilon}^{2} \int_{\Omega} \psi\left|\partial_{t} u_{\varepsilon}\right|^{2}- & \frac{\alpha_{\varepsilon}^{2}}{\alpha_{\varepsilon}^{2}+1} \int_{\Omega} \nabla \psi \cdot\left(f_{\varepsilon}, \nabla u_{\varepsilon}\right) \\
& \quad+\frac{\alpha_{\varepsilon}}{\alpha_{\varepsilon}^{2}+1}(\nabla \psi+\mathbb{J} \nabla \phi) \cdot\left(i f_{\varepsilon}, \nabla u_{\varepsilon}\right) .
\end{aligned}
$$

Proof. Summing (3.8) and (3.13), we obtain

$$
\begin{aligned}
& \partial_{t} \int_{\Omega}\left(\alpha_{\varepsilon} \psi e_{\varepsilon}\left(u_{\varepsilon}\right)+\phi J\left(u_{\varepsilon}\right)\right) \\
& \quad=-\alpha_{\varepsilon}^{2} \int_{\Omega} \psi\left|\partial_{t} u_{\varepsilon}\right|^{2}+\alpha_{\varepsilon} \int_{\Omega} \psi \operatorname{div}\left(\partial_{t} u, \nabla u\right)+\int_{\Omega} \phi \operatorname{curl}\left(i \partial_{t} u, \nabla u\right) .
\end{aligned}
$$

Using that $\partial_{t} u_{\varepsilon}=\frac{1}{\alpha_{\varepsilon}+i} f_{\varepsilon}$, we have that

$$
\left(\partial_{t} u_{\varepsilon}, \nabla u_{\varepsilon}\right)=\left(\frac{\alpha_{\varepsilon}-i}{\alpha_{\varepsilon}^{2}+1} f_{\varepsilon}, \nabla u_{\varepsilon}\right)
$$

and

$$
\left(i \partial_{t} u_{\varepsilon}, \nabla u_{\varepsilon}\right)=\left(\frac{1+i \alpha_{\varepsilon}}{\alpha_{\varepsilon}^{2}+1} f_{\varepsilon}, \nabla u_{\varepsilon}\right),
$$

so we can rewrite (3.15) by decomposing the right-hand side in terms of $\left(f_{\varepsilon}, \nabla u_{\varepsilon}\right)$ and $\left(i f_{\varepsilon}, \nabla u_{\varepsilon}\right)$, yielding

$$
\begin{aligned}
& \text { (3.16) } \partial_{t} \int_{\Omega}\left(\alpha_{\varepsilon} \psi e_{\varepsilon}\left(u_{\varepsilon}\right)+\phi J\left(u_{\varepsilon}\right)\right) \\
& =-\alpha_{\varepsilon}^{2} \int_{\Omega} \psi\left|\partial_{t} u_{\varepsilon}\right|^{2}+\frac{1}{\alpha_{\varepsilon}^{2}+1} \int_{\Omega} \phi \operatorname{curl}\left(f_{\varepsilon}, \nabla u_{\varepsilon}\right)+\frac{\alpha_{\varepsilon}^{2}}{\alpha_{\varepsilon}^{2}+1} \int_{\Omega} \psi \operatorname{div}\left(f_{\varepsilon}, \nabla u_{\varepsilon}\right) \\
& \quad+\frac{\alpha_{\varepsilon}}{\alpha_{\varepsilon}^{2}+1}\left(-\psi \operatorname{div}\left(i f_{\varepsilon}, \nabla u_{\varepsilon}\right)+\phi \operatorname{curl}\left(i f_{\varepsilon}, \nabla u_{\varepsilon}\right)\right) .
\end{aligned}
$$

We note that our choices of signs are such that $\int \eta \operatorname{curl} A=\int A \cdot \mathbb{J} \nabla \eta$ for functions $\eta$ and vector fields $A$. Integrating the terms in (3.16) by parts and using (3.6), we obtain the claim.

We will choose test functions that are affine and satisfy $\nabla \psi+\mathbb{J} \nabla \phi=0$ near the vortex core. A possible choice is $\psi(x)=x_{1}$ and $\phi(x)=-x_{2}$. Then the last two lines of (3.14) can be controlled, and the rest converges to one component of the motion law. 
As in [1], [7, Lemma 2.3.1], and [17], we can use the following relation between the stress-energy tensor of the canonical harmonic map and the gradient of the renormalized energy:

Proposition 3.2. Let $\phi \in W_{0}^{2, \infty}(\Omega)$ such that for some $i \in\{1, \ldots, n\}$, $\phi$ is affine in $B_{\rho}\left(a_{i}\right)$, and $\phi=0$ in $B_{\rho}\left(a_{j}\right)$ for $j \neq i$. Then we have for $u_{*}=u_{*}^{(a, d)}$

$$
\begin{aligned}
\int_{\Omega} \mathbb{J}_{k l} \partial_{k m} \phi\left(j\left(u_{*}\right)_{m} j\left(u_{*}\right)_{l}\right) & \\
& =\mathbb{J} \nabla \phi\left(a_{i}\right) \cdot \nabla_{a_{i}} W(a, d)=-\nabla \phi \cdot \mathbb{J} \nabla_{a_{i}} W(a, d) .
\end{aligned}
$$

Proof. We set $b=\mathbb{J} \nabla \phi\left(a_{i}\right)$ and note that $\mathbb{J} \nabla \phi(x) \equiv b$ in $B_{\rho}\left(a_{i}\right)$. For $s<\frac{1}{2} \rho_{a}$, the function

$$
v_{*}^{s}(x)=u_{*}(x+s \rrbracket \nabla \phi(x))
$$

now satisfies

$$
\operatorname{curl} j\left(v_{*}^{s}\right)=2 \pi \sum_{j \neq i} d_{j} \delta_{a_{j}}+2 \pi d_{i} \delta_{a_{i}-s b} .
$$

We set $a^{s}=\left(a_{1}, \ldots, a_{i}-s b, \ldots, a_{n}\right)$ and compare, using the minimality property, the energy of $v_{*}^{s}$ with the renormalized energy corresponding to the moved vortex:

$$
2 W\left(a^{s}, d\right) \leq \lim _{r \rightarrow 0}\left(\int_{\Omega_{r}\left(a^{s}\right)}\left|\nabla v_{*}^{s}\right|^{2}-\pi \log \frac{1}{r}\right) .
$$

Subtracting (2.8), we obtain

$$
\frac{1}{s}\left[W\left(a^{s}\right)-W(a)\right] \leq \frac{1}{2 s}\left(\int_{\Omega_{r}\left(a^{s}\right)}\left|\nabla v_{*}^{s}\right|^{2}-\int_{\Omega_{r}(a)}\left|\nabla u_{*}\right|^{2}\right)
$$

for $r$ sufficiently small. Note that we do not need to take limits as $v_{*}^{s}(x-s b)=$ $u_{*}(x)$ in $B_{\rho}(a)$.

By change of variables and using that $\mathbb{J} \nabla \phi$ is divergence free, we can calculate

$$
\left.\frac{d}{d s}\right|_{s=0} \int_{\Omega_{r}\left(a^{s}\right)}\left|\nabla v_{*}^{s}\right|^{2}=\int_{\Omega} \mathbb{J}_{i k} \partial_{j} \partial_{k} \phi\left(\partial_{i} u_{*}, \partial_{j} u_{*}\right),
$$

so comparing with (3.21), we obtain

$$
-b \cdot \nabla_{a_{i}} W(a, d) \leq \int_{\Omega} \mathbb{J}_{i k} \partial_{j} \partial_{k} \phi\left(\partial_{i} u_{*}, \partial_{j} u_{*}\right) .
$$

Applying the same argument to the function $v_{*}^{-s}$ and $W\left(a^{-s}\right)$ yields the opposite inequality, and since $\left(\partial_{l} u_{*}, \partial_{m} u_{*}\right)=j\left(u_{*}\right)_{l} j\left(u_{*}\right)_{m}$, the proposition follows by renaming indices.. 


\section{Results on the vortex Paths}

In this section, we assume that $\left(u_{\varepsilon}\right)$ is a sequence of solutions to (1.1)-(1.2) such that the well-preparedness conditions (2.15) and (2.16) hold. We note that throughout the rest of the paper, $C$ will denote constant independent of $\varepsilon$ and $t$.

Proposition 4.1. For $0 \leq t<T_{0}$, the energy is bounded by the initial energy,

$$
E_{\varepsilon}\left(u_{\varepsilon}(\cdot, t)\right) \leq E_{\varepsilon}\left(u_{\varepsilon}^{0}\right) .
$$

Furthermore, there exists $\bar{T}>0$ such that the kinetic energy bound

$$
\int_{0}^{\bar{T}} \int_{\Omega}\left|\partial_{t} u_{\varepsilon}\right|^{2} d x d t \leq \frac{C}{\alpha_{\varepsilon}}
$$

holds. The right-hand side $f_{\varepsilon}$ can be estimated by

$$
\int_{0}^{\bar{T}} \int_{\Omega}\left|f_{\varepsilon}\right|^{2} d x d t \leq C \frac{\alpha_{\varepsilon}^{2}+1}{\alpha_{\varepsilon}} \leq \frac{2 C}{\alpha_{\varepsilon}}
$$

for $\varepsilon$ sufficiently small.

Proof. Using (3.13), we see that after multiplying (1.1) with $\partial_{t} u$ and integrating over $(0, s) \times \Omega$, there holds

$$
\alpha_{\varepsilon} \int_{0}^{s} \int_{\Omega}\left|\partial_{t} u_{\varepsilon}\right|^{2}=-E_{\varepsilon}\left(u_{\varepsilon}(s)\right)+E_{\varepsilon}\left(u_{\varepsilon}(0)\right) .
$$

We can now follow the argument of [21, Lemma 3.4] to deduce the existence of a time $\bar{T}$ such that (4.2) holds, since otherwise, it would be possible to find (by suitable rescalings of time) a time $t_{\varepsilon} \ll 1$ such that the vortices do not move until $t_{\varepsilon}$, but the energy decreases by at least $O(1)$, which leads to a contradiction between the assumption of initial well-preparedness (2.16) and the $\Gamma$-lim inf inequality (2.9). The bound (4.3) then follows by noting that $f_{\varepsilon}=\left(\alpha_{\varepsilon}+i\right) \partial_{t} u_{\varepsilon}$ by $(1.1)$.

Proposition 4.2. For every time $t \in[0, \bar{T}]$, the Jacobians $J\left(u_{\varepsilon}(t)\right)$ converge in $\left(C_{0}^{0, \beta}\right)^{*}$ to a measure $\mu(t)$ that can be written as

$$
\mu(t)=\pi \sum_{i=1}^{n} d_{i} \delta_{\xi_{i}(t)},
$$

and the points $\xi_{i}$ satisfy $\xi_{i} \in H^{1}(0, \bar{T} ; \Omega)$. Furthermore, for all $0 \leq t_{1}<$ $t_{2}<\bar{T}$,

$$
\liminf _{\varepsilon \rightarrow 0} \alpha_{\varepsilon} \int_{\Omega \times\left(t_{1}, t_{2}\right)}\left|\partial_{t} u_{\varepsilon}\right|^{2} \geq \pi \alpha_{0} \sum_{i=1}^{n} \int_{t_{1}}^{t_{2}}\left|\dot{\xi}_{i}\right|^{2} .
$$

In particular the vortex paths are $C^{0,1 / 2}$ continuous in time. 
Proof. These results are shown in Propositions 3.2, 3.3, and the proof of Lemma 3.4 of [21]. The mobility bound (4.6) is given in Corollary 7 of $[22]$.

We always let $a(t)$ denote the solution of the ODE system (2.17) with initial conditions $a(0)=a^{0}=\xi(0)$. To understand how much the energy of the ODE motion deviates from the PDE energy, we introduce the energy excess

$$
D_{\varepsilon}(t)=E_{\varepsilon}\left(u_{\varepsilon}(t)\right)-W_{\varepsilon}(a(t), d)
$$

and the corresponding limiting energy excess

$$
D(t)=\limsup _{\varepsilon \rightarrow 0} D_{\varepsilon}(t) .
$$

We also let $\eta(t) \in \mathbb{R}^{2 n}$ denote the difference between the vortices found by Proposition 4.2 and the solutions of the ODE:

$$
\eta_{i}=\xi_{i}-a_{i}, i=1, \ldots, n .
$$

Proposition 4.3. For all $0 \leq t_{1}<t_{2}<T$, the energy excess satisfies

$$
D_{\varepsilon}\left(t_{2}\right)-D_{\varepsilon}\left(t_{1}\right) \leq o_{\varepsilon}(1)-\pi \alpha_{0} \sum_{i=1}^{n} \int_{t_{1}}^{t_{2}}\left|\dot{\eta}_{i}\right|^{2}-2 \pi \alpha_{0} \sum_{i=1}^{n} \int_{t_{1}}^{t_{2}} \dot{a}_{i} \dot{\eta}_{i} .
$$

Proof. Differentiating $\frac{d}{d t} W(a(t), d)=\dot{a} \cdot \nabla_{a} W(a(t), d)$ and using (4.4),

$$
\begin{aligned}
D_{\varepsilon}\left(t_{2}\right) & =E_{\varepsilon}\left(u_{\varepsilon}\left(t_{2}\right)\right)-W_{\varepsilon}\left(a\left(t_{2}\right), d\right) \\
& =E_{\varepsilon}\left(u_{\varepsilon}\left(t_{2}\right)\right)-E_{\varepsilon}\left(u_{\varepsilon}\left(t_{1}\right)\right)+W_{\varepsilon}\left(a\left(t_{1}\right), d\right)-W_{\varepsilon}\left(a\left(t_{2}\right), d\right)+D_{\varepsilon}\left(t_{1}\right) \\
& =-\alpha_{\varepsilon} \int_{t_{1}}^{t_{2}} \int_{\Omega}\left|\partial_{t} u_{\varepsilon}\right|^{2}-\int_{t_{1}}^{t_{2}} \sum_{i=1}^{n} \dot{a}_{i} \cdot \nabla_{a_{i}} W(a(t), d)+D_{\varepsilon}\left(t_{1}\right) .
\end{aligned}
$$

Using that $a(t)$ satisfies $(2.17), \dot{a}_{i} \cdot \nabla_{a_{i}} W(a(t), d)=-\pi \alpha_{0}\left|\dot{a}_{i}\right|^{2}$. By (4.4) and $(4.6)$,

$$
\begin{aligned}
D_{\varepsilon}\left(t_{2}\right)-D_{\varepsilon}\left(t_{1}\right) & =-\alpha_{\varepsilon} \int_{t_{1}}^{t_{2}}\left|\partial_{t} u_{\varepsilon}\right|^{2}+\pi \alpha_{0} \int_{t_{1}}^{t_{2}} \sum_{i=1}^{n}\left|\dot{a}_{i}\right|^{2} \\
& \leq o_{\varepsilon}(1)-\pi \alpha_{0} \int_{t_{1}}^{t_{2}} \sum_{i=1}^{n}\left(\left|\dot{\xi}_{i}\right|^{2}-\left|\dot{a}_{i}\right|^{2}\right) .
\end{aligned}
$$

Now $\xi_{i}+a_{i}=\xi_{i}-a_{i}+2 a_{i}=\eta_{i}+2 a_{i}$, and so (4.10) follows.

We need to be able to estimate $|\eta(t)|$. To this end, we define the ODE errors

$$
R_{i}=\alpha_{0} \dot{\xi}_{i}-d_{i} \rrbracket \dot{\xi}_{i}+\frac{1}{\pi} \nabla_{a_{i}} W(a, d),
$$

so

$$
\alpha_{0} \dot{\xi}_{i}-d_{i} \rrbracket \dot{\xi}_{i}=-\frac{1}{\pi} \nabla_{a_{i}} W(a, d)+R_{i}
$$


We obtain the following evolution for the error in vortex positions $\eta_{i}$ :

$$
\alpha_{0} \dot{\eta}_{i}-d_{i} \mathbb{J} \dot{\eta}_{i}=R_{i},
$$

so taking the norm on both sides,

$$
\sqrt{\alpha_{0}^{2}+1}\left|\dot{\eta}_{i}\right|=\left|R_{i}\right|
$$

We can combine these estimates into

Proposition 4.4. The growth rate of the energy excess and the vortex position error is controlled by the equation error. More precisely, for every $T$ smaller than the collision time for (2.17), there is a constant $C=C(a(0), T)$ such that for every $0<t_{1} \leq t_{2}<T$,

$$
D_{\varepsilon}\left(t_{2}\right)+|\eta|\left(t_{2}\right) \leq D_{\varepsilon}\left(t_{1}\right)+|\eta|\left(t_{1}\right)+o_{\varepsilon}(1)+C \int_{t_{1}}^{t_{2}}|R(t)| .
$$

Proof. From (4.10) and (4.14), we see that we only need to bound $\sup _{t}|\dot{a}|$, which is controlled by the ODE.

Proposition 4.5. If $D_{\varepsilon}(t) \ll|\log \varepsilon|$ and

$$
\left\|J\left(u_{\varepsilon}\right)-\pi \sum_{i=1}^{n} d_{i} \delta_{\xi_{i}}\right\|_{\dot{W}^{-1,1}} \rightarrow 0,
$$

then also the rescaled energy density converges to a limit supported at the same points:

$$
\left\|\frac{\alpha_{\varepsilon}}{\alpha_{0}} e_{\varepsilon}\left(u_{\varepsilon}\right)-\pi \sum_{i=1}^{n} \delta_{\xi_{i}}\right\|_{\dot{W}^{-1,1}} \rightarrow 0 .
$$

Proof. This is essentially shown (with an estimate on the convergence speed) in Theorem 1.4.4 of [7].

\section{Bounds InVOLVing THE ENERGY EXCESS}

In this section, we establish some estimates on the error terms that will be useful in the following section. We always assume that $t_{0}$ is a time such that $D_{\varepsilon}\left(t_{0}\right)=o(1), \eta\left(t_{0}\right)=0$, and $\tau$ is so small that (4.3) holds.

As our main tool, we use the following somewhat simplified version of the refined estimates of [12]:

Proposition 5.1. There exist constants $\varepsilon_{0}>0, C>0$ and $K>1$ depending only on $n$ and $\Omega$ with the following property: Assume $u \in H^{1}(\Omega)$ with $E_{\varepsilon}(u) \leq C|\log \varepsilon|$ such that there exist $a \in \Omega^{n}$ with $\rho_{a}>0$ and $d \in\{ \pm 1\}^{n}$ with

$$
\left\|J(u)-\pi \sum_{i=1}^{n} d_{i} \delta_{a_{i}}\right\|_{\dot{W}^{-1,1}} \leq s .
$$


Then if $4 s \leq \frac{\rho_{a}}{4 K}, \sigma=\frac{\rho_{a}}{2}$, and $\varepsilon<\varepsilon_{0}$ the energy on $\Omega_{\sigma}(a)$ satisfies the bounds

$$
\int_{\Omega_{\sigma}(a)} e_{\varepsilon}(|u|)+\frac{1}{4}\left|\frac{j(u)}{|u|}-j\left(u_{*}^{(a, d)}\right)\right|^{2} \leq E_{\varepsilon}(u)-W_{\varepsilon}(a, d)+C\left(\frac{s+\varepsilon^{1 / 2}}{\rho_{a}}\right)
$$

and

$$
\begin{aligned}
& \left\|j(u)-j\left(u_{*}^{(a, d)}\right)\right\|_{L^{4 / 3}(\Omega)} \\
& \quad \leq C \sqrt{E_{\varepsilon}(u)-W_{\varepsilon}(a, d)}+C\left(\frac{s+\varepsilon^{1 / 2}}{\rho_{a}}\right)\left(\rho_{a}^{-1 / 4}+1\right) .
\end{aligned}
$$

Here our u satisfies either (1.3) or (1.4) on $\partial \Omega$.

Proof. This result is a consequence of Theorem 2 in [12], and can be obtained by choosing (in the notation of (8.8) of [12]) $\sigma=\sqrt{\rho_{\alpha}\left(s+\varepsilon^{1 / 2}\right)}$ and then reducing the domain of integration to $\Omega \frac{\rho_{a}}{2}(a)$.

We note that Theorem 2 of [12] was proved for the case of Neumann boundary conditions; however, the proof of this theorem can be modified rather easily to handle Dirichlet boundary conditions. In fact the only change that needs to be made is to justify the integration by parts argument for $A_{1}$ in the proof of Lemma 4 of [12]. We point out, however, that the boundary integral disappears (as in the Neumann boundary condition case), since the Dirichlet boundary conditions imply $\int_{\partial \Omega} G_{\sigma} \tau \cdot\left(j(u)-j\left(u_{*}\right)\right)=0$, where $G_{\sigma}$ is the conjugate harmonic map of the limiting current $j\left(u_{*}\right)$.

We apply this to solutions of (1.1) to obtain

Proposition 5.2. If $|\eta(t)| \leq \frac{\sigma}{4 K}$ for $t \in\left[t_{0}, t_{0}+\delta\right]$, then

$$
\alpha_{\varepsilon} \int_{t_{0}}^{t_{0}+\delta} \int_{\Omega_{\sigma}\left(a\left(t_{0}\right)\right)}\left|\left(i f_{\varepsilon}, \nabla u_{\varepsilon}\right)\right| \leq C \sqrt{\alpha_{\varepsilon}} \sqrt{|\log \sigma|+\int_{t_{0}}^{t_{0}+\delta} D_{\varepsilon}} .
$$

Proof. Using the Cauchy-Schwarz inequality, we have

$$
\begin{aligned}
& \int_{t_{0}}^{t_{0}+\delta} \int_{\Omega_{\sigma}\left(a\left(t_{0}\right)\right)}\left|\left(i f_{\varepsilon}, \nabla u_{\varepsilon}\right)\right| \\
& \quad \leq\left(\int_{t_{0}}^{t_{0}+\delta} \int_{\Omega_{\sigma}\left(a\left(t_{0}\right)\right)}\left|f_{\varepsilon}\right|^{2}\right)^{1 / 2}\left(\int_{t_{0}}^{t_{0}+\delta} \int_{\Omega_{\sigma}\left(a\left(t_{0}\right)\right)}\left|\nabla u_{\varepsilon}\right|^{2}\right)^{1 / 2} .
\end{aligned}
$$

Since $\left|\xi(t)-a\left(t_{0}\right)\right| \leq \frac{\sigma}{2}$, we obtain for every $t$ from (5.1), using $\left|\nabla u_{\varepsilon}\right|^{2}=$ $\left.|\nabla| u_{\varepsilon}\right|^{2}+\left|\frac{j\left(u_{\varepsilon}\right)}{\left|u_{\varepsilon}\right|}\right|^{2}$ and an easy bound on $j\left(u_{*}^{(a(t), d)}\right)$. In particular

$$
\int_{\Omega_{\sigma}\left(a\left(t_{0}\right)\right)}|\nabla u|^{2} \leq C|\log \sigma|+D_{\varepsilon}+C|\eta|
$$


and hence (4.3) yields the result.

Proposition 5.3. For any $\delta \leq \tau$,

$$
\frac{\alpha_{\varepsilon}^{2}}{\alpha_{\varepsilon}^{2}+1} \int_{t_{0}}^{t_{0}+\delta} \int_{\Omega}\left|\left(f_{\varepsilon}, \nabla u_{\varepsilon}\right)\right|=o(1) \quad \text { as } \varepsilon \rightarrow 0 .
$$

Proof. Again by Cauchy-Schwarz and using (4.3),

$$
\begin{aligned}
\int_{t_{0}}^{t_{0}+\delta} \int_{\Omega}\left|\left(f_{\varepsilon}, \nabla u_{\varepsilon}\right)\right| & \left.\leq\left(\int_{t_{0}}^{t_{0}+\delta} \int_{\Omega}\left|f_{\varepsilon}\right|^{2}\right)^{1 / 2}\left(\int_{t_{0}}^{t_{0}+\delta} \int_{\Omega}\left|\nabla u_{\varepsilon}\right|^{2}\right)\right)^{1 / 2} \\
& \leq \frac{C}{\sqrt{\alpha_{\varepsilon}}} \sqrt{\delta}|\log \varepsilon|^{1 / 2} \leq C
\end{aligned}
$$

and since $\alpha_{\varepsilon} \rightarrow 0$ we obtain the claim.

Proposition 5.4. For every $\delta \leq \tau$ such that $D_{\varepsilon}(t) \leq C$ and $|\eta(t)| \leq \frac{\sigma}{4 K}$ for $t \in\left[t_{0}, t_{0}+\delta\right]$, we have the estimate

$$
\begin{array}{r}
\int_{t_{0}}^{t_{0}+\delta} \mid \int_{\Omega} \mathbb{J}_{k l} \partial_{k m} \phi \\
\quad\left(\left(\partial_{m} u, \partial_{l} u\right)-\left(j\left(u_{*}\right)\right)_{m}\left(j\left(u_{*}\right)\right)_{l}\right) \mid \\
\leq C \int_{t_{0}}^{t_{0}+\delta} D_{\varepsilon}(t)+|\eta|(t)+o_{\varepsilon}(1) .
\end{array}
$$

Proof. We use the decomposition of $\nabla u \otimes \nabla u$ as used in [12]. By orthogonality, we have that $\nabla u=\nabla|u| \frac{u}{|u|}+i \frac{j(u)}{|u|} \frac{u}{|u|}$, so after substituting $\frac{j(u)}{|u|}=j\left(u_{*}\right)+\left(\frac{j(u)}{|u|}-j\left(u_{*}\right)\right)$, where $u_{*}=u_{*}^{(a(t), d)}$,

$$
\begin{aligned}
\left.\int_{\Omega} \mathbb{J}_{k l} \partial_{k m} \phi\left(\left(\partial_{m} u, \partial_{l} u\right)-\left(j\left(u_{*}\right)\right)_{m} j\left(u_{*}\right)\right)_{l}\right) \\
=\int_{\Omega} \mathbb{J}_{k l} \partial_{k m} \phi \partial_{m}|u| \partial_{l}|u| \\
\quad+\int_{\Omega} \mathbb{J}_{k l} \partial_{k m} \phi\left(\frac{j(u)}{|u|}-j\left(u_{*}\right)\right)_{m}\left(\frac{j(u)}{|u|}-j\left(u_{*}\right)\right)_{l} \\
\quad+\int_{\Omega} \mathbb{J}_{k l} \partial_{k m} \phi\left(\frac{j(u)}{|u|}-j\left(u_{*}\right)\right)_{m}\left(j\left(u_{*}\right)\right)_{l} \\
\quad+\int_{\Omega} \mathbb{J}_{k l} \partial_{k m} \phi\left(j\left(u_{*}\right)\right)_{m}\left(\frac{j(u)}{|u|}-j\left(u_{*}\right)\right)_{l} .
\end{aligned}
$$

We estimate these terms one by one. First of all, we note that

$$
\int_{\Omega} \mathbb{J}_{k l} \partial_{k m} \phi \partial_{m}|u| \partial_{l}|u| \leq \frac{C}{\sigma^{2}} \int_{\Omega_{\sigma}}|\nabla| u||^{2} \leq \frac{C}{\rho_{*} \sigma^{2}}(|\eta(t)|+o(1))
$$

as in (3.24) of [12].

To estimate the next term,

$$
\int_{\Omega} \mathbb{J}_{k l} \partial_{k m} \phi\left(\frac{j(u)}{|u|}-j\left(u_{*}\right)\right)_{m}\left(\frac{j(u)}{|u|}-j\left(u_{*}\right)\right)_{l},
$$


we use Proposition 5.1, which shows that this term is bounded by $\frac{C}{\sigma^{2}}\left(D_{\varepsilon}+\right.$ $|\eta|+o(1))$. For the remaining terms, it suffices by symmetry to consider one of them. We have

$$
\begin{aligned}
\int_{\Omega} \mathbb{J}_{k l} \partial_{k m} \phi\left(\frac{j(u)}{|u|}-j\left(u_{*}\right)\right)_{m}\left(j\left(u_{*}\right)\right)_{l} \\
=\int_{\Omega} \mathbb{J}_{k l} \partial_{k m} \phi\left(\frac{j(u)}{|u|}-j(u)\right)_{m}\left(j\left(u_{*}\right)\right)_{l} \\
\quad+\int_{\Omega} \mathbb{J}_{k l} \partial_{k m} \phi\left(j(u)-j\left(u_{*}\right)\right)_{m}\left(j\left(u_{*}\right)\right)_{l}
\end{aligned}
$$

We note that $\frac{j(u)}{|u|}-j(u)$ can be estimated as

$$
\left|\frac{j(u)}{|u|}-j(u)\right|=\left|\frac{j(u)}{|u|}(1-|u|)\right| \leq|\nabla u||1-| u||
$$

so the first term on the RHS of (5.8) is bounded by

$$
\begin{aligned}
\left\|D^{2} \phi\right\|_{L^{\infty}}\left\|\nabla u_{*}\right\|_{L^{\infty}}\|\nabla u\|_{L^{2}} \| & -|u|^{2} \|_{L^{2}} \\
& \leq C(\sigma) \varepsilon|\log \varepsilon|=o_{\varepsilon}(1) .
\end{aligned}
$$

For the second term, we perform a spatial Hodge decomposition of $j(u)-$ $j\left(u_{*}\right)$ for every time $t$. In the Neumann boundary condition case (1.4) we write $j(u)-j\left(u_{*}\right)=\nabla h_{1}(t)+\mathbb{J} \nabla h_{2}(t)$ where $h_{1}(t) \in W^{1, p}(\Omega)$ is the solution of

$$
\Delta h_{1}(t)=\operatorname{div} j(u(t)), \quad \nu \cdot \nabla h_{1}(t)=0 \text { on } \partial \Omega
$$

and $h_{2}(t) \in W^{1, p}(\Omega)$ solves

$$
\Delta h_{2}(t)=\operatorname{curl}\left(j(u(t))-j\left(u_{*}^{(a(t), d)}\right)\right), \quad h_{2}(t)=0 \text { on } \partial \Omega .
$$

Note that in the case of Dirichlet boundary conditions (1.3), our $h_{1}(t)$ and $h_{2}(t)$ solve the same equations except $h_{1}(t)=\nu \cdot \nabla h_{2}(t)=0$ on $\partial \Omega$. A similar analysis can be done on this Hodge decomposition as the analysis found below.

We continue with our analysis of (5.10) and (5.11). Setting $\zeta_{m}(t)=$ $\mathbb{J}_{k l} \partial_{k m} \phi\left(j\left(u_{*}^{(a(t), d)}\right)\right)_{l}$, then it follows that

$$
\int_{\Omega} \zeta(t) \cdot\left(j(u)-j\left(u_{*}\right)\right)=\int_{\Omega} \zeta(t) \cdot\left(\nabla h_{1}(t)+\mathbb{J} \nabla h_{2}(t)\right) .
$$

We claim that

$$
\left|\int_{t_{0}}^{t_{0}+\tau} \int_{\Omega} \zeta \cdot \nabla h_{1} d x d t\right| \rightarrow 0
$$

as $\varepsilon \rightarrow 0$. In order to show (5.13) we first note from (5.2) that for each time, $\left\|j(u)-j\left(u_{*}\right)\right\|_{L^{4 / 3}(\Omega)} \leq C$. This implies by virtue of $L^{p}$ theory for the Hodge decomposition that $\left\|\nabla h_{1}(t)\right\|_{L^{4 / 3}(\Omega)} \leq C$ for all $t$, which in turn implies that $\nabla h_{1} \in L^{4 / 3}\left(\left[t_{0}, t_{0}+\tau\right] \times \Omega\right)$. Hence, the sequence of $h_{1}$ 's are 
weakly compact. According to Proposition 5.5 below, the only possible limit must be 0 . Since $\zeta \in L^{\infty}\left(\left[t_{0}, t_{0}+\tau\right] \times \Omega\right)$, then the estimate (5.13) holds.

For the remaining term, $\int_{t_{0}}^{t_{0}+\tau}\left|\int_{\Omega} \zeta(t) \cdot \mathbb{J} \nabla h_{2}(t)\right|$, we use that $\dot{W}^{-1,1}$ embeds into $\dot{W}^{-1, p}$. In particular since $\Delta h_{2}=J(u)-\pi d_{j} \delta_{a_{j}}$, then by standard elliptic estimates (see [20]) we see $\left\|h_{2}\right\|_{W^{1, p}(\Omega)} \leq C\left\|J(u)-\pi d_{j} \delta_{a_{j}}\right\|_{\dot{W}^{-1, p}(\Omega)} \leq$ $C\left\|J(u)-\pi d_{j} \delta_{a_{j}}\right\|_{\dot{W}^{-1,1}(\Omega)} \leq C|\eta|+o_{\varepsilon}(1)$. Therefore,

$$
\left\|\mathbb{J} \nabla h_{2}(t)\right\|_{L^{p}(\Omega)} \leq C|\eta(t)|+o_{\varepsilon}(1) .
$$

Combining (5.7), (5.8), (5.9), (5.12), (5.13), and (5.14), we obtain (5.6).

In the proof above, we have used the following result, which is a consequence of the conservation of mass equation:

Proposition 5.5. The divergence $\operatorname{div} j\left(u_{\varepsilon}\right)$ tends to zero in the dual space of $W_{0}^{1,4}(\Omega \times(0, T))$. In particular

$$
\left|\int_{\Omega \times(0, T)} \nabla \phi \cdot j\right| \leq C\|\phi\|_{W^{1,4}((0, T) \times \Omega)}\left(\sqrt{\alpha_{\varepsilon}}+\varepsilon \sqrt{|\log \varepsilon|}\right)
$$

for all $\phi \in W_{0}^{1,4}(\Omega \times(0, T))$.

Proof. It follows from the conservation of mass equation (3.9) that

$$
\operatorname{div} j(u)=\alpha_{\varepsilon}\left(i u, \partial_{t} u\right)+\frac{1}{2} \partial_{t}\left(|u|^{2}-1\right),
$$

where we have written $u$ for $u_{\varepsilon}$. Let $\phi \in W_{0}^{1,4}$. Then

$$
\begin{gathered}
\int_{0}^{T} \int_{\Omega} \phi \operatorname{div} j(u)=\alpha_{\varepsilon} \int_{0}^{T} \int_{\Omega} \phi\left(i u, \partial_{t} u\right)+\frac{1}{2} \int_{0}^{T} \int_{\Omega} \phi \partial_{t}\left(|u|^{2}-1\right) \\
\leq \alpha_{\varepsilon}\left(\int_{0}^{T} \int_{\Omega}|\phi|^{4}\right)^{1 / 4}\left(\int_{0}^{T} \int_{\Omega}|u|^{4}\right)^{1 / 4}\left(\int_{0}^{T} \int_{\Omega}\left|\partial_{t} u\right|^{2}\right)^{1 / 2} \\
-\left.\frac{1}{2} \int_{0}^{T} \int_{\Omega} \partial_{t} \phi|| u\right|^{2}-1 \mid \\
\leq C \sqrt{\alpha_{\varepsilon}}\|\phi\|_{L^{4}((0, T) \times \Omega)}+C \varepsilon \sqrt{|\log \varepsilon|}\left\|\partial_{t} \phi\right\|_{L^{4}((0, T) \times \Omega)} \\
\leq C\|\phi\|_{W^{1,4}((0, T) \times \Omega)}\left(\sqrt{\alpha_{\varepsilon}}+\varepsilon \sqrt{|\log \varepsilon|}\right) \rightarrow 0 \text { as } \varepsilon \rightarrow 0 .
\end{gathered}
$$

\section{Proof of the main theorem}

To prove Theorem 2.2, we first state a result that is local in time. We then show that the local result implies the global result, and finally give a proof of the local version.

Proposition 6.1. Starting at a time $t_{0}<T$ with $D_{\varepsilon}\left(t_{0}\right)=o(1)$, there exists $a \tau_{0}$ such that the conclusions of Theorem 2.2 are valid on $\left[t_{0}, t_{0}+\tau_{0}\right)$. 
Proof of Theorem 2.2. Following Sandier-Serfaty [21], we let $\tilde{T}$ be the supremum over all times such that the conclusions of Theorem 2.2 hold. By Proposition $6.1, \tilde{T}>0$. Assume now by contradiction that $\tilde{T}<T$. We will show that Proposition 6.1 can be applied at $\tilde{T}$.

For every $t<\tilde{T}, \xi(t)=a(t)$ and the energy excess vanishes, so (4.4) implies

$$
\alpha_{\varepsilon} \int_{0}^{t} \int_{\Omega}\left|\partial_{t} u_{\varepsilon}\right|^{2}=-E_{\varepsilon}\left(u_{\varepsilon}(0)\right)+E_{\varepsilon}\left(u_{\varepsilon}(t)\right) \rightarrow-W(a(t), d)+W(a(0), d) .
$$

As $\tilde{T}$ is before the ODE collision time, the right-hand side of (6.1) is bounded as $t \rightarrow \tilde{T}$, and so the kinetic energy bound (4.2) holds until time $\tilde{T}$. From [22, Theorem 3], we obtain the existence of continuous vortex paths $\xi(t)$ on $[0, \tilde{T}]$. The continuity of $\xi$ and $a$ in time implies $\xi(\tilde{T})=a(\tilde{T})$.

Using that

$$
E_{\varepsilon}\left(u_{\varepsilon}(\tilde{T})\right) \leq E_{\varepsilon}\left(u_{\varepsilon}(t)\right) \leq n(\pi|\log \varepsilon|+\gamma)+W(a(t), d)+o(1),
$$

for all $t \leq \tilde{T}$,

$$
\left.\limsup _{\varepsilon \rightarrow 0}\left(E_{\varepsilon}\left(u_{\varepsilon}(\tilde{T})\right)-n(\pi|\log \varepsilon|+\gamma)\right) \leq W(a(t), d)\right) \rightarrow W(a(\tilde{T}), d),
$$

so $D_{\varepsilon}(\tilde{T})=o(1)$ and so Proposition 6.1 can be applied to contradict the maximality of $\tilde{T}$.

Proof of Proposition 6.1. From Proposition 4.1, we find a $\bar{\tau}<T-t_{0}$ such that

$$
\int_{t_{0}}^{t_{0}+\bar{\tau}} \int_{\Omega}\left|\partial_{t} u_{\varepsilon}\right|^{2} \leq \frac{C}{\alpha_{\varepsilon}}
$$

We set

$$
A_{*}=\max _{t_{0} \leq t \leq t_{0}+\bar{\tau}}|\dot{a}(t)|
$$

and

$$
\rho_{*}=\min _{t_{0} \leq t \leq t_{0}+\bar{\tau}} \rho_{a(t)}
$$

With $\sigma=\frac{\rho_{*}}{2}$ and $K$ denoting the constant in Proposition 5.1, we define $\tau=\min \left(\bar{\tau}, \frac{\sigma}{4 K A_{*}}\right)$. It follows that $a_{i}(t) \in B_{\sigma / 4 K}\left(a_{i}\left(t_{0}\right)\right)$ for all $t \in\left[t_{0}, t_{0}+\tau\right]$. Fixing a function $\chi \in C_{0}^{\infty}\left(\mathbb{R}^{2}\right)$ with $\chi=1$ in $B_{1}(0), \chi=0$ outside $B_{2}(0)$, we define $\chi_{\sigma}(x)=\chi\left(\frac{x}{\sigma}\right)$. 
We want to apply $(3.14)$ to $(\psi, \phi)=\left(\psi_{1}^{(i)}, \phi_{1}^{(i)}\right)$ and $(\psi, \phi)=\left(\psi_{2}^{(i)}, \phi_{2}^{(i)}\right)$ given by

$$
\begin{aligned}
& \psi_{1}^{(i)}=x_{1} \chi_{\sigma}\left(x-a_{i}\left(t_{0}\right)\right), \\
& \phi_{1}^{(i)}=-x_{2} \chi_{\sigma}\left(x-a_{i}\left(t_{0}\right)\right), \\
& \psi_{2}^{(i)}=x_{2} \chi_{\sigma}\left(x-a_{i}(0)\right), \\
& \phi_{2}^{(i)}=x_{1} \chi_{\sigma}\left(x-a_{i}(0)\right) .
\end{aligned}
$$

The main feature of these choices of $(\psi, \phi)$ is that

$$
\nabla \psi_{j}^{(i)}+\mathbb{J} \nabla \phi_{j}^{(i)}=0 \text { in } B_{\sigma}\left(a_{i}\left(t_{0}\right)\right), \quad j=1,2 .
$$

Furthermore, we have the bound

$$
\left|\nabla \phi_{j}^{(i)}\right|+\left|\nabla \psi_{j}^{(i)}\right| \leq \frac{C}{\sigma} \text { in } B_{2 \sigma}\left(a_{i}\left(t_{0}\right)\right) \backslash B_{\sigma}\left(a_{i}\left(t_{0}\right)\right) .
$$

In the following, we will drop the sub- and superscripts on $\psi$ and $\phi$ for convenience if the results are valid for every $i$ and $k$.

We use (3.17) and rewrite

$$
\begin{aligned}
& -\nabla \phi\left(a_{i}\right) \cdot \mathbb{J} \nabla_{a_{i}} W(a, d) \\
= & -\frac{\alpha_{\varepsilon}^{2}}{\alpha_{\varepsilon}^{2}+1} \nabla \phi\left(a_{i}\right) \cdot \mathbb{J} \nabla_{a_{i}} W(a, d)+\frac{1}{\alpha_{\varepsilon}^{2}+1} \int_{\Omega} \mathbb{J}_{k l} \partial_{k m} \phi\left(j\left(u_{*}\right)\right)_{m}\left(j\left(u_{*}\right)\right)_{l} .
\end{aligned}
$$

We thus obtain from (3.14)

$$
\begin{gathered}
{\left[\left(\partial_{t} \int_{\Omega} \psi \alpha_{\varepsilon} e_{\varepsilon}\left(u_{\varepsilon}\right)+\phi J\left(u_{\varepsilon}\right)\right)-\nabla \phi\left(a_{i}\right) \cdot \mathbb{J} \nabla_{a_{i}} W(a, d)\right]} \\
=-\alpha_{\varepsilon}^{2} \int_{\Omega} \psi\left|\partial_{t} u_{\varepsilon}\right|^{2}-\frac{\alpha_{\varepsilon}^{2}}{\alpha_{\varepsilon}^{2}+1} \int_{\Omega} \nabla \psi \cdot\left(f_{\varepsilon}, \nabla u_{\varepsilon}\right) \\
+\frac{\alpha_{\varepsilon}}{\alpha_{\varepsilon}^{2}+1} \int_{\Omega}(\nabla \psi+\mathbb{J} \nabla \phi) \cdot\left(i f_{\varepsilon}, \nabla u_{\varepsilon}\right)-\frac{\alpha_{\varepsilon}^{2}}{\alpha_{\varepsilon}^{2}+1} \nabla \phi\left(a_{i}\right) \cdot \mathbb{J} \nabla_{a_{i}} W(a, d) \\
+\frac{1}{\alpha_{\varepsilon}^{2}+1}\left(\int_{\Omega} \mathbb{J}_{k l} \partial_{k m} \phi\left(\partial_{m} u, \partial_{l} u\right)-\int_{\Omega} \mathbb{J}_{k l} \partial_{k m} \phi\left(j\left(u_{*}\right)\right)_{m}\left(j\left(u_{*}\right)\right)_{l}\right),
\end{gathered}
$$

and so we obtain by taking absolute values and integrating in time,

$$
\begin{aligned}
& \int_{t_{0}}^{t_{0}+\delta} \mid \partial_{t}\left[\int_{\Omega} \psi \alpha_{\varepsilon} e_{\varepsilon}\left(u_{\varepsilon}\right)+\phi J\left(u_{\varepsilon}\right)\right]- \nabla \phi\left(a_{i}\right) \cdot \mathbb{J} \nabla_{a_{i}} W(a, d) \mid \\
& \leq A_{1}+A_{2}+A_{3}+A_{4}+A_{5},
\end{aligned}
$$


where

$$
\begin{aligned}
& A_{1}=\alpha_{\varepsilon}^{2} \int_{t_{0}}^{t_{0}+\delta} \int_{\Omega}|\psi|\left|\partial_{t} u_{\varepsilon}\right|^{2}, \\
& A_{2}=\frac{\alpha_{\varepsilon}^{2}}{\alpha_{\varepsilon}^{2}+1} \int_{t_{0}}^{t_{0}+\delta} \int_{\Omega}\left|\nabla \psi \cdot\left(f_{\varepsilon}, \nabla u_{\varepsilon}\right)\right| \\
& A_{3}=\frac{\alpha_{\varepsilon}}{\alpha_{\varepsilon}^{2}+1} \int_{t_{0}}^{t_{0}+\delta} \int_{\Omega}\left|(\nabla \psi+\mathbb{J} \nabla \phi) \cdot\left(i f_{\varepsilon}, \nabla u_{\varepsilon}\right)\right|, \\
& A_{4}=\frac{\alpha_{\varepsilon}^{2}}{\alpha_{\varepsilon}^{2}+1} \int_{t_{0}}^{t_{0}+\delta}\left|\nabla \phi\left(a_{i}\right) \cdot \mathbb{J} \nabla_{a_{i}} W(a, d)\right|, \\
& A_{5}=\frac{1}{\alpha_{\varepsilon}^{2}+1} \int_{t_{0}}^{t_{0}+\delta} \mid \int_{\Omega} \mathbb{J}_{k l} \partial_{k m} \phi\left(\left(\partial_{m} u, \partial_{l} u\right)-\left(j\left(u_{*}\right)\right)_{m}\left(j\left(u_{*}\right)_{l}\right) \mid .\right.
\end{aligned}
$$

It is immediate that $A_{4}=o(1)$. By (4.2), $A_{1}=o(1)$ if $D_{\varepsilon}\left(t_{0}\right)=o(1)$ and $\delta<\bar{T}$. By Propositions 5.2 and 5.3, we control $A_{2}$ and $A_{3}$. Finally, the bound for $A_{5}$ is given in Proposition 5.4, so

$$
A_{1}+A_{2}+A_{3}+A_{4}+A_{5} \leq C \int_{t_{0}}^{t_{0}+\delta} D_{\varepsilon}(t)+|\eta(t)|+o(1)
$$

The convergence (Propositions 4.2, 4.5) of $\alpha_{\varepsilon} e_{\varepsilon}\left(u_{\varepsilon}\right)$ and of $J\left(u_{\varepsilon}\right)$ imply the weak convergence

$$
\partial_{t} \int_{\Omega} \psi \alpha_{\varepsilon} e_{\varepsilon}\left(u_{\varepsilon}\right)+\phi J\left(u_{\varepsilon}\right) \rightarrow \pi \partial_{t}\left(\alpha_{0} \psi\left(\xi_{i}\right)+d_{i} \phi\left(\xi_{i}\right)\right),
$$

where the right-hand side can be written as

$$
\pi \partial_{t}\left(\alpha_{0} \psi\left(\xi_{i}\right)+d_{i} \phi\left(\xi_{i}\right)\right)=\pi\left(\alpha_{0} \nabla \psi\left(\xi_{i}\right)+d_{i} \nabla \phi\left(\xi_{i}\right)\right) \cdot \dot{\xi}_{i}
$$

Now the definitions of $\phi$ and $\psi$ imply

$$
\nabla \psi_{1}^{(i)}\left(\xi_{i}\right)=\left(\begin{array}{l}
1 \\
0
\end{array}\right), \quad \nabla \psi_{2}^{(i)}\left(\xi_{i}\right)=\left(\begin{array}{l}
0 \\
1
\end{array}\right), \quad \nabla \phi_{1}^{(i)}\left(\xi_{i}\right)=\left(\begin{array}{c}
0 \\
-1
\end{array}\right), \quad \nabla \phi_{2}^{(i)}\left(\xi_{i}\right)=\left(\begin{array}{l}
1 \\
0
\end{array}\right)
$$

so, writing the right hand sides of (6.10) for fixed $i$ and $k=1,2$ as a single vector, we obtain $\pi\left(\alpha_{0} \dot{\xi}_{i}-d_{i} \mathbb{J} \dot{\xi}_{i}\right)$. We also note that

$$
\nabla_{a_{i}} W(a, d)=-\left(\begin{array}{c}
\nabla \phi_{1}^{(i)}\left(a_{i}\right) \cdot \mathbb{J} \nabla_{a_{i}} W(a, d) \\
\nabla \phi_{2}^{(i)}\left(a_{i}\right) \cdot \mathbb{J} \nabla_{a_{i}} W(a, d)
\end{array}\right) .
$$

We thus obtain from (6.7), using the weak convergence (6.9) and the lower semicontinuity of the $L^{1}$ norm that we can bound the equation error $R_{i}=$ $\alpha_{0} \dot{\xi}_{i}-d_{i} \mathbb{J} \dot{\xi}_{i}+\frac{1}{\pi} \nabla_{a_{i}} W(a, d)$ by the limit of (6.8), so

$$
\int_{t_{0}}^{t_{0}+\delta}\left|R_{i}\right| \leq C \liminf _{\varepsilon \rightarrow 0} \int_{t_{0}}^{t_{0}+\delta}\left(D_{\varepsilon}(t)+|\eta(t)|+o(1)\right),
$$

and now combining this and Proposition 4.4, we see by Gronwall's inequality that $R(t)=0$ and so also $\eta(t)=0$ and $\lim \sup D_{\varepsilon}(t)=0$ for all $t \in\left[t_{0}, t_{0}+\delta\right]$. 
(Note that technically, we can only bound some of the $A_{i}$ if $|\eta|+D_{\varepsilon}$ is small enough; however, this can be always assumed by taking $\delta$ sufficiently small).

The fact that $\eta(t)=0$ establishes the motion law; $\lim \sup D_{\varepsilon}(t)=0$ gives us the propagation of well-preparedness, and so the proof is complete.

\section{REFERENCES}

[1] Bethuel, F., Brezis, H., and Hélein, F. Ginzburg-Landau vortices. Birkhäuser, Boston 1994.

[2] Brezis, H., Coron, J.-M., and Lieb, E. Harmonic maps with defects. Comm. Math. Phys. 107 (1996), 649-705.

[3] Bethuel, F., Jerrard, R., and Smets, D. On the NLS dynamics for infinite energy vortex configurations in the plane. Rev. Mat. Iberoamericana 24 (2008), 671-702.

[4] Bethuel, F., Orlandi, G., and Smets, D. Collisions and phase-vortex interactions in dissipative Ginzburg-Landau dynamics. Duke Math. J. 130 (2005), 523-614.

[5] Bethuel, F., Orlandi, G., and Smets, D. Quantization and motion law for GinzburgLandau vortices. Arch. Ration. Mech. Anal. 183 (2007), 315-370.

[6] Colliander, J., and Jerrard, R. Vortex dynamics for the Ginzburg-Landau-Schrödinger equation. Internat. Math. Res. Notices 7 (1998), 333-358.

[7] Colliander, J., and Jerrard, R. Ginzburg-Landau vortices: weak stability and Schrödinger equation dynamics. Journal d'Analyse Mathématique 77 (1999), 129 205.

[8] Dorsey, A. Vortex motion and the Hall effect in type II superconductors: a timedependent Ginzburg-Landau approach. Phys. Rev. B 46 (1992), 8376-8392.

[9] E, W. Dynamics of vortices in Ginzburg-Landau theories with applications to superconductivity. Physica D 77 (1994), 383-404.

[10] Hang, F., and Lin, F., Static theory for planar ferromagnets and antiferromagnets. Acta Math. Sinica, English Series 17 (2001), 541-580.

[11] Jerrard, R., and Soner, H. Dynamics of Ginzburg-Landau vortices. Arch. Rational Mech. Anal. 142 (1998), 99-125.

[12] Jerrard, R., and Spirn, D. Refined Jacobian Estimates and Gross-Pitaevsky Vortex Dynamics. Arch. Rational Mech. Anal. 190 (2008), 425-475.

[13] Kurzke, M., Melcher, C., Moser, R., and Spirn, D., in preparation.

[14] Lin, F., Some dynamical properties of Ginzburg-Landau vortices. Comm. Pure Appl. Math. 49 (1996), 323-359.

[15] Lin, F., A remark on the previous paper: "Some dynamical properties of GinzburgLandau vortices" Comm. Pure Appl. Math. 49 (1996), 361-364.

[16] Lin, F., and Shatah, J. Soliton dynamics in planar ferromagnets and antiferromagnets. Journal of Zhejiang University Science 4 (2003), 503-510.

[17] Lin, F., Xin, J. On the incompressible fluid limit and the vortex motion law of the nonlinear Schrödinger equation. Comm. Math. Phys 200 (1999), 249-274.

[18] Miot, E. Dynamics of vortices for the complex Ginzburg-Landau equation. Preprint arxiv:0810.4782, 2008.

[19] Neu, J. Vortices in complex scalar fluids. Physica D 43 (1990), 385-406.

[20] Roitberg, Y. Elliptic Boundary Value Problems in the Space of Distributions, Mathematics and its Applications. Kluwer Academic Publications, Boston, (1999).

[21] Sandier, E., and Serfaty, S. Gamma convergence of gradient flows with applications to Ginzburg-Landau. Comm. Pure Appl. Math. 57 (2004), 1627-1672.

[22] Sandier, E., and Serfaty, S. A product estimate for Ginzburg-Landau and corollaries. J. Funct. Anal. 211 (2004), 219-244.

[23] Serfaty, S. Vortex collisions and energy-dissipation rates in the Ginzburg-Landau heat flow. II. The dynamics. J. Eur. Math. Soc. (JEMS) 9 (2007), 383-426. 
DYNAMICS FOR GINZBURG-LANDAU VORTICES UNDER A MIXED FLOW 21

[24] Serfaty, S. Local minimizers for the Ginzburg-Landau energy near critical magnetic field. I. Comm. Contemp. Math. 1 (1999), 213-254.

[25] Serfaty, S. Local minimizers for the Ginzburg-Landau energy near critical magnetic field. II. Comm. Contemp. Math. 1 (1999), 295-333.

Institut Für Angewandte Mathematik, Wegelerstrasse 10, D-53115 Bonn, GeRmany

E-mail address: kurzke@iam.uni-bonn.de

Mathematical Institute, 24-29 St Giles', Oxford OX1 3LB, United Kingdom

E-mail address: melcher@maths.ox.ac.uk

Department of Mathematical Sciences, University of Bath, Bath BA2 7AY, UNITED KINGDOM

E-mail address: r.moser@bath.ac.uk

School of Mathematics, University of Minnesota, Minneapolis, MN 55455, USA

E-mail address: spirn@math.umn.edu 